\title{
Development of Moringa oleifera Standardized Leaf Extract Nanofibers Impregnated onto Hydrocolloid Film as A Potential Chronic Wound Dressing
}

\author{
Chai-Yee Chin and Shiow-Fern Ng* \\ Centre for Drug Delivery Research, Faculty of Pharmacy, Universiti Kebangsaan Malaysia, \\ Jalan Raja Muda Abdul Aziz, Kuala Lumpur 50300, Malaysia \\ (Received December 16, 2019; Revised February 19, 2020; Accepted February 25, 2020)
}

\begin{abstract}
This research focuses on the development of a hybrid dressing, i.e. Moringa oleifera (MOL) leave extract nanofibers impregnated onto an alginate-pectin hydrocolloid film for chronic wound healing. The MOL nanofibers impregnated hydrocolloid films were characterised and optimised by physicomechanical properties, moisture transmission, swelling ratio, microscopy analysis, Franz diffusion drug release and cell viability assays. From the preformulation studies, PEO $6 \%$ solution with viscosity above $1.3 \mathrm{~Pa} \cdot \mathrm{s}$ was found suitable to be electrospun into uniform nanofibers (diameter $322.7 \pm 78.02 \mathrm{~nm}$ ). The moisture transmission rate and swelling ratio studies revealed that the MOL nanofiber-film was useful for the application of light to medium suppurating wounds. The Franz diffusion cell study showed that the electrospun duration could influence the amount of MOL released from the nanofibers-film. Finally, cell viability assays revealed that the MOL nanofibers-films hybrid dressing did not cause cytotoxicity to human dermal fibroblast and human epithelial keratinocytes cell lines. In conclusion, a hybrid MOL nanofibers impregnated hydrocolloid films dressing was successfully formulated and this study has implied that MOL nanofibers-film could be an alternative treatment option for treating chronic wounds.
\end{abstract}

Keywords: Moringa oleifera, Wound healing, Electrospun nanofibers, Hydrocolloid film, Alginate, Pectin

\section{Introduction}

Diabetic foot ulcer (DFUs) is one of the common delayed chronic wounds among diabetic patients worldwide. As per world statistics as updated by WHO (World Health Organisation) in 2017, it was estimated 415 million increased up to 612 million of adult will have diabetes in next coming 25 years [1]. The current treatment of delayed chronic wound is still ineffective, as chronic wound requires a wound dressing which can provide a moist environment, reduce pain and release actives at a sustain release manner to accelerate wound healing. Conventional dressings such as gauze, creams, or hydrogels cannot perform as such.

Nanofibers have been used in the biomedical applications for the past two decades. Nanofibers have become an important part of biomedical research and they are intensively studied for tissue scaffold technology, food packaging and drug delivery system for wound healing [26]. Electrospinning nanofibers are polymer fibres ranging from nanometre to micrometres diameter produced at high voltage electrical field. It has characteristics such as (1) high porosity, (2) high surface to volume ratio, (3) biomimetic for good biocompatibility with skin, (4) improved cell proliferation property for drug-delivery and (5) anti-haemostatic wound application [7]. The nanofiber provides excellent nanofibrous 3dimensional extracellular matrix support to various specialised cells for growth. Hence it is an ideal platform for wound delivery of therapeutic agents. Currently, various types of polymers were studied to produce nanofibers for wounds

*Corresponding author: nsfern@ukm.edu.my especially delayed chronic or burn wounds, these include polyvinyl alcohol (PVA), polyacrylonitrile (PAN), cellulose acetate, chitosan and polyethylene oxide (PEO) [8-13]. PEO is a biodegradable and non-toxic hydrophilic polymer which has been widely utilised in nanofiber fabrication process. Due to the high hydrophilicity of PEO, it formed a homogeneous solution with MOL aqueous extract and suitable for electrospinning. Hence, PEO with high molecular weight $(900 \mathrm{kDa})$, was selected in nanofiber formulation in order to produce a smooth uniform MOL extract nanofiber. However, it forms a thin mat without cross-linking, causing it is to be too delicate to handle. This problem could be overcome by impregnating the nanofibers onto a hydrocolloid thin film as a protective backing layer to prolong the application duration of the wound dressing.

Moringa oleifera leaf (MOL) extract had been used by traditional folks for wound and skin sores for centuries [1416]. Until recently, there were several in vitro and in vivo studies had been conducted with MOL extracts alone [1719]. The findings revealed that it possesses an excellent antiinflammatory property and helps in faster wound epithelisation and collagen deposition [20,21]. And yet it still lacks studies on incorporating MOL extract into nanofiber impregnated film dressing for wound healing application. MOL can be highly potential wound healing agent by incorporating into nanofibers and become new application by combining hydrocolloid film with nanofiber into a wound dressing.

This research focuses on the development of Moringa oleifera leaf aqueous extract into nanofibers through electrospinning technique and combination of nanofiber with alginate-pectin based hydrocolloid film into a hybrid 
wound dressing for potential delayed chronic wound healing application. It also followed by several parameters of material characterisations such as SEM imaging, moisture vapour transmission rate (MVTR), swelling ratio and in vitro drug release studies. Our laboratory is the first to successfully load MOL onto electrospun nanofibers for wound application. The advantage of using nanofibers as carrier can helps to deliver MOL effectively onto wound site due to the large contact area with wound tissues. This could provide a new insight in developing plant extract nanofiber based wound dressing for accelerated wound healing.

\section{Experimental}

\section{Materials}

For hybrid nanofiber-film fabrication, poly-(ethylene oxide) (PEO) (CAS No. 25322-68-3) with average molecular weight $900 \mathrm{kDa}$, and sodium alginate (CAS No. 9005-38-3) were procured from Sigma-Aldrich (UK). Pectin (CAS No.9000-69-5) and glycerol (CAS No.56-81-5) were purchased from R\&M Chemicals (UK). Moringa oleifera leaves (MOL) were collected from Kuala Terengganu between October and November month of 2016. The leaf samples were authenticated with specimen voucher UKMB40408 as deposited in herbarium of Universiti Kebangsaan Malaysia (UKM). MOL aqueous extract was prepared by using cold maceration technique followed by lyophilisation process.

\section{Blank and MOL Loaded Extract-Loaded Nanofibers}

The protocol of nanofiber fabrication via electrospinning method was adapted from Krstic et al. [10] with slight modification. PEO $(4-6 \% \mathrm{w} / \mathrm{w})$ was dissolved in sterile deionised water and stirred homogeneously with magnetic stirrer at $80^{\circ} \mathrm{C}$ for 60 mins. The solutions were then allowed to cool down to ambient temperature $\left(27 \pm 3{ }^{\circ} \mathrm{C}\right)$. Before electrospinning, the polymer blend solutions were introduced into $1 \mathrm{ml}$ syringe capped to 23 -gauge stainless blunt tip needle. The blunt tip needle attached with a voltage alligator clip to high-voltage power model (Gamma High Voltage Research, USA) while the one connected to electrical grounded metal collector. The metal collector was covered with aluminium foil and used as nanofiber collecting area. During electrospinning process, the applied electrostatic voltage power was kept at $20 \mathrm{kV}$ and flow rate of blend solutions kept at $1 \mathrm{ml} / \mathrm{h}$ controlled by automatic syringe pump (New Era Pumps System, USA). The polymer blend solutions also electrospun at horizontal tip-to-collector distance $(15 \mathrm{~cm})$ to the metal collector for $15 \mathrm{~min}$ to produce a thin nanofiber mat. The whole process of electrospinning was performed at room temperature $\left(27 \pm 3{ }^{\circ} \mathrm{C}\right)$. The formed nanofibers (F7-F9) were observed under light microscope (Olympus, Japan) at magnification x200 for fibre morphology. Hence the nanofiber polymer formulations which produced the most satisfactory nanofibers were incorporated with $1 \%$ MOL extracts. The MOL aqueous extract was prepared according to our previous work (Chin et al. 2017). To produce MOL extract nanofibers, the MOL extract was added into polymer blend solution before electrospinning (N1-N3).

\section{Characterisation on Electrospun Nanofibers}

The blank and MOL extract-loaded nanofibers were further characterised using light and variable pressure scanning electron microscope (VPSEM) for its detailed fibre morphology and diameter measurements. Whereas the rheology study was performed on the blank and MOL extractloaded polymer blend solutions to assess the influence of polymer solution viscosity to the formation of the nanofiber.

Variable Pressure Scanning Electron Microscope (VPSEM)

After the preliminary nanofiber formation observation using light microscope at 200x magnication, the blank nanofibers and MOL extract-loaded nanofibers were visualised under VPSEM. For VPSEM, firstly the nanofibers were sputter-coated with palladium for $30 \mathrm{~s}$ before visualised by VPSEM at magnification $5 \mathrm{k}$ and $10 \mathrm{kX}$. The images obtained from VPSEM were analysed by using image $\mathrm{J}$ software (1.50i version). Three images were taken for each nanofiber formulation and at least ten fibres in each image were randomly selected for average fibre diameter measurement.

\section{Rheology Study}

The rheology study was performed to measure the dynamic viscosity profiles of the most satisfactory PEO nanofibers with or without MOL extracts using Bohlin Gemini Rheometer (Malvern Instruments, UK) in accordance to the protocols established by Thu et al. [23] but with slight modifications. Approximately $1 \mathrm{~m} l$ volume of each polymer blend solution was placed on the Peltier plate before measured with the cone and plate system which is $20 \mathrm{~mm}$ diameter with an angle of $2^{\circ}$. The shear rate was set to increase from zero to $300 \mathrm{~s}^{-1}$ within 3 mins and dropped back to zero again with constant rate in the same time interval. The average apparent viscosities of each sample were obtained at shear rate of $300 \mathrm{~s}^{-1}$. All measurements conducted in triplicate and the average mean value was computed. The average apparent viscosity values of each polymer blend solution were compared at a shear rate of $300 \mathrm{~s}^{-1}$.

\section{MOL Loaded Nanofibers-Hydrocolloid Film}

The MOL nanofibers were electrospun onto the backing layer which was alginate-pectin (SA-PC) hydrocolloid film. To prepare the backing layer, distilled water was firstly prewarmed at temperature $50 \pm 5^{\circ} \mathrm{C}$ and mixed homogeneously with $3 \mathrm{~g}$ of glycerol. It was then followed by addition of these polymers (sodium alginate and pectin) 


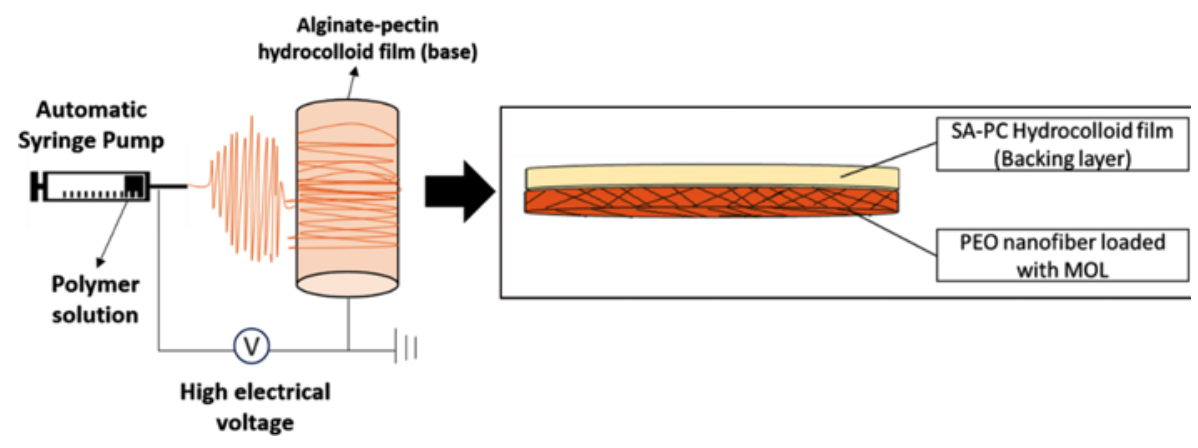

Figure 1. Schematic images of electrospinning process to MOL loaded nanofiber-hydrocolloid film dressing.

(3\% w/v respectively) and constantly stirred with magnetic stirrer $(500 \mathrm{rpm})$ until complete dissolution. The polymer blend solution $(25 \mathrm{~g})$ was casted carefully to flat bottomed petri dish (90 $\mathrm{mm}$ diameter). It was then developed into dried SA-PC thin film after dried in the oven at temperature $40 \pm 4{ }^{\circ} \mathrm{C}$ for 24 hours. To this end, formulation N3 appeared as the most satisfactory nanofiber after fibre morphology visualisation under light microscope and VPSEM. The formulation N3 was electrospun onto the SA-PC hydrocolloid film. The entire process was the same as described in nanofiber fabrication protocol, except that the nanofibers were electrospun onto hydrocolloid film instead of aluminium foil on the receiver (Figure 1).

Characterisation of MOL Loaded Nanofibers-Hydrocolloid Film

The MOL-loaded nanofiber-hydrocolloid film dressing was then characterised in terms of physicochemical properties such as swelling ratio and moisture vapour transmission rate (MVTR), in comparison to blank SA-PC vehicle film alone and blank nanofiber-film.

\section{Expansion Studies}

The expansion properties of blank SA-PC vehicle film, blank nanofiber-film, and MOL-loaded nanofiber-film were measured by using established wound stimulating fluid model with slight modifications [24]. The wound simulating fluid was prepared using $4 \% \mathrm{w} / \mathrm{v}$ gelatine powder (Type B, Sigma-Aldrich, UK) dissolved in distilled water. The gelatine solution was heated gradually at $50{ }^{\circ} \mathrm{C}$ for $15 \mathrm{mins}$ and stirred with magnetic stirrer to achieve complete dissolution. It was then followed by $20 \mathrm{~g}$ of gelatine solution cast on petri dish and incubated at room temperature overnight. The nanofiber samples were moulded into $22 \mathrm{~mm}$ circular disc and located in the centre of gelatine solutions. The expansion profile was recorded by measuring the expansion diameter for first subsequent 8 hours and 24th hour. The measurements were repeated triplicate. The average value was computed by using following equation (1):

Film swelling ratio $(\%)=\left(D_{t}-D_{0}\right) / D_{0} \times 100 \%$

where, $D_{t}$ is diameter of film after expansion, $D_{0}$ is diameter of film before expansion.

Moisture Vapour Transmission Rate (MVTR)

MVTR study was performed to measure the gaseous exchange among blank SA-PC vehicle film, blank nanofiberfilm, and MOL-loaded nanofiber-film. The films were shaped into circular form $(\mathrm{d}=22 \mathrm{~mm})$ before sealed with rubber cap in clear glass vials. The vials contain $2 \mathrm{~g}$ of dry silica beads as desiccant with nanofibers were weighed as 0 hour. The MVTR study was conducted at first subsequent 8 hours, followed by 24 th and 48 th hour in moist chamber with calcium chloride solution in saturated state $\mathrm{RH}$ $80 \pm 3 \%$ ). The weight gains of vials for respective hours were recorded and triplicate measurements were taken to obtain the average weight gain. Hence, MVTR was computed using the following equation (2):

$$
\text { Moisture vapour transmission rate }(\mathrm{MVTR})=\mathrm{W} / \mathrm{S}
$$

where, $W$ is the weight gain of the silica bead over $24 \mathrm{~h}$ and $S$ is the exposed surface area of the film $\left(\mathrm{m}^{2}\right)$, MVTR expressed in unit as $\mathrm{g} / \mathrm{m}^{2} /$ day.

Optimisation of MOL Loaded Nanofibers-Hydrocolloid Film

After nanofiber formulation studies and characterisations, the MOL-loaded nanofiber formulation (N3) was selected as the most desirable nanofiber due to its uniform fibre without droplets and beads formation. N3 was further subjected for various electrospinning duration at 15, 30 and $60 \mathrm{~min}$ on the hydrocolloid film. The resulted nanofiber-films were characterised for macroscopic appearance, mechanical property, moisture absorption and in vitro drug release.

Macroscopic Surface Morphology of Fabricated MOLloaded Nanofiber Films

The most satisfactory nanofiber (N3) formulation had electrospun on the SA-PC thin hydrocolloid film for respective time durations $(15,30$ and $60 \mathrm{~min})$. The resulted nanofiber-films were captured by digital camera (Samsung WB10, South Korea) for macroscopic surface observation.

\section{Mechanical Properties}

The mechanical properties of MOL-loaded nanofiberfilms were characterised in accordance to ASTM guidelines 
D882-02 using universal testing machine (Instron, USA). The nanofiber-films were moulded into $30 \mathrm{~mm}$ long and $4 \mathrm{~mm}$ width dumbbell-shaped ribbons by using ASTM standard mould. The samples were stretched at crosshead speed of $5 \mathrm{~mm} / \mathrm{min}$ to its rupturing point to measure tensile strength $(\mathrm{mPa})$ and elongation at break (\%). The samples were tested in triplicate and mean values were calculated based on the following equations (3) and (4):

$$
\begin{aligned}
& \text { TS }(\mathrm{MPa})=\text { Max force }(\mathrm{N}) / \text { thickness of film }(\mathrm{mm}) \\
& \times \text { width of film }(\mathrm{mm})
\end{aligned}
$$

where, TS is tensile strength, expressed in unit as $\mathrm{mPa}(\mathrm{N} /$ $\mathrm{mm}^{2}$ ).

$$
\text { Elongation at break }(\% \mathrm{E})=L / L_{0} \times 100 \%
$$

where, $L$ is extension length of film at rupturing point, $L_{0}$ is initial length of film before extension $(30 \mathrm{~mm})$.

\section{Moisture Absorption Study}

The nanofiber-film was shaped into $22 \mathrm{~mm}$ circular disc and kept in desiccator with silica beads before moisture absorption testing. The nanofiber-film samples were weighed with microbalance and recorded as $0 \mathrm{~h}$, before transferred to a closed moist chamber with saturated calcium chloride $(\mathrm{RH}$ $80 \%$ ). The weight gains of sample were measured after $24 \mathrm{~h}$ in the moist chamber and the measurements were repeated in triplicate. The moisture absorption percentage was computed by the following equation (5):

$$
\text { Moisture absorption } \%=\left(W-W_{0}\right) / W_{0} \times 100 \%
$$

where, $W$ is the weight of nanofiber-film after $24 \mathrm{~h}$, and $W_{0}$ is the initial weight of nanofiber-film before transferred to the moist chamber.

In vitro Drug Release Study via Franz Diffusion Cells

MOL-loaded nanofiber-films (15, 30 and 60 mins) were subjected for in vitro drug release study via Franz diffusion cells (Permegear Inc., USA) in accordance to established protocol by Thu et al. [23]. The Franz diffusion receptor compartment filled up with phosphate buffer saline (PBS) $(5.1 \pm 0.1 \mathrm{~m} l)$ which acts as receptor medium. A $13-\mathrm{mm}$ diameter synthetic cellulose acetate filter membrane $(0.45 \mu \mathrm{m}$ pore size) was placed on the top receptor orifice and followed by nanofiber-film $(13 \mathrm{~mm})$ clipped between donor orifice. The cumulative drug release study was performed for first $8 \mathrm{~h}$ with $2 \mathrm{~h}$ interval and 24th h. In each time point, $0.5 \mathrm{~m} l$ of receptor medium was collected through donor compartment and refilled with equal amount of fresh PBS solution. The drug release aliquot samples from MOL loaded nanofiber-films (15, 30 and $60 \mathrm{~min})$ were analysed using high performance liquid chromatography (HPLC) respectively.

Quantification of Bioactive Compounds by HPLC Technique

The sample aliquots from Franz diffusion cells were subjected to separation and quantification using Waters reverse phase (RP) high performance liquid chromatography
(HPLC) system coupled with a photodiode array detector (2998) and an autosampler (2707) via gradient mode. The stationary phase consists of C18 analytical column $(250 \mathrm{~mm}$ long $\times 4.6 \mathrm{~mm}$ interior diameter, $5 \mu \mathrm{m}$ particle size) (Waters XBridge). The HPLC mobile phase consists of (A) $0.1 \%$ TFA in ultrapure water and (B) acetonitrile. The HPLC gradient mode was conducted rapidly for $8 \mathrm{~min}$ as follows: 0.01-4 min, 10-95 \% B; 4-6 min, $95 \%$ B; 6-6.1 min, 95-10 \% $\mathrm{B}$; and re-equilibration to initial conditions for $2 \mathrm{~min}$. The column was kept at $35{ }^{\circ} \mathrm{C}$ with constant flow rate kept at $0.5 \mathrm{ml} / \mathrm{min}$. The sample volume of injected into the HPLC system was $20 \mu l$.

There were three reference standards (chlorogenic acid, rosmarinic acid, and vicenin-2) to set up an external standard calibration curve with concentration ranged from $0.5,0.2$, $0.1,0.02$ and $0.01 \mathrm{mg} / \mathrm{l}$. The obtained peak areas of each standards were integrated using Empower chromatographic software interfaced to a computer.

In Vitro Biocompatibility Assay

In order to assess the status of biocompatibility of nanofiber embedded film at various electrospinning durations (15, 30 and $60 \mathrm{~min})$, each sample was tested with human dermal fibroblast (HDF) and epidermal keratinocytes (HEK) cell line. The nanofiber embedded films were trimmed to circular disc $(\mathrm{d}=10 \mathrm{~mm})$ and deposited into the 24-wells plate before UV irradiation for an hour. After UV irradiation for sterilization, each well was seeded with HDF (ATCC, USA) at cell density $0.5 \times 10^{5}$ at a volume of $100 \mu l$ and left incubated in a controlled-temperature incubator $\left(37^{\circ} \mathrm{C}, 5 \%\right.$ $\mathrm{CO}_{2}$ ) overnight for $24 \mathrm{~h}$. The in-vitro biocompatibility of nanofiber embedded films was assessed by commercially available MTT (3-(4,5-Dimethylthiazol-2-yl)-2,5-Diphenyltetrazolium Bromide) cell counting kit. After $24 \mathrm{~h}$ incubation, $50 \mu l$ of MTT solution $(5 \mathrm{mg} / \mathrm{m} l)$ was added into each well followed by incubation for $4 \mathrm{~h}$ in the incubator at temperature $37 \pm 2{ }^{\circ} \mathrm{C}$. The MTT formazan formed at the bottom of the 24-wells plate was dissolved with $200 \mu l$ of dimethyl sulfoxide (DMSO) solution after the spent media discarded. The dissolved MTT formazan $(100 \mu l)$ ) was transferred carefully to 96-wells plate before absorbance of each well read at $570 \mathrm{~nm}$ wavelength with a microplate reader. The well which contains cell suspension treated with culture media acts as untreated control while the well with cell suspension treated with PEO nanofiber acts as vehicle control. The percentage of cell viability of each well was computed by the following equation:

$$
\begin{aligned}
& \text { Cell viability }(\%)=(\text { Sample OD } 570 \mathrm{~nm}-\text { Untreated OD } \\
& 570 \mathrm{~nm}) / \text { Untreated OD } 570 \mathrm{~nm} \times 100 \%
\end{aligned}
$$

where, cell viability beyond $80 \%$ is considered non-toxic, in accordance to the ISO 10993-5:2009 guideline.

\section{Statistical Analysis}

All test data were presented as mean $\pm S D(n=3)$ and 
evaluated using one-way analysis of variance (ANOVA) by Graph Pad Prism. For one-way ANOVA, the $p$ value $<0.05$ was indicated as statistically significant threshold for the differences between the groups.

\section{Results and Discussion}

\section{Blank and MOL Extract-Loaded Nanofibers}

Nanofibers were fabricated via electrospinning method and morphology observations were summarised in Table 1 and Figure 2. F7-F9 which composed by PEO alone were successfully electrospun into smooth nanofibers with minimal beads observed. The formulations F7-F9 were further incorporated with $1 \% \mathrm{w} / \mathrm{v}$ Moringa oleifera standardised leaf (MOL) extract. And the formulations were given codes

Table 1. Morphology of electrospun nanofiber during preformulations

\begin{tabular}{|c|c|c|c|}
\hline $\begin{array}{l}\text { Formulation } \\
\text { code }\end{array}$ & $\begin{array}{l}\text { PEO } \\
\text { conc. } \\
\text { (wt } \%)\end{array}$ & $\begin{array}{l}\text { MOL } \\
\text { conc. } \\
\text { (wt } \%)\end{array}$ & Nanofiber appearance \\
\hline F7 & 4 & - & $\begin{array}{l}\text { Non-homogeneous nanofiber with } \\
\text { beads appearance }\end{array}$ \\
\hline F8 & 5 & - & $\begin{array}{l}\text { Smooth and uniform nanofiber } \\
\text { formed }\end{array}$ \\
\hline F9 & 6 & - & $\begin{array}{l}\text { Non-homogeneous nanofiber with } \\
\text { beads appearance }\end{array}$ \\
\hline N1 & 4 & 1 & $\begin{array}{l}\text { Non-homogeneous nanofiber with } \\
\text { beads }\end{array}$ \\
\hline $\mathrm{N} 2$ & 5 & 1 & Smooth and uniform nanofiber formed \\
\hline N3 & 6 & 1 & Smooth and uniform nanofiber formed \\
\hline
\end{tabular}

$\mathrm{N} 1, \mathrm{~N} 2$ and $\mathrm{N} 3$ for 4,5 and $6 \%$ PEO respectively.

\section{Blank and MOL Loaded Electrospun Nanofibers Microscopy Observations}

The light microscopic observation, Figure 3 revealed that N1 \& N2 had electrospun to nanofibers with spindle-like beads. Only the formulation PEO $6 \% \mathrm{w} / \mathrm{v}$ blend (N3) with MOL extract could be electrospun to uniform nanofiber without droplets and beads. In the process of fabricating nanofibers, it is important that good quality nanofiber strands, i.e. smooth, uniform and continuous in appearance are produced. Irregularity, discontinuous strings or the presence bead-like structures in the fibre strands are not desirable features of nanofibers. The blank PEO nanofibers

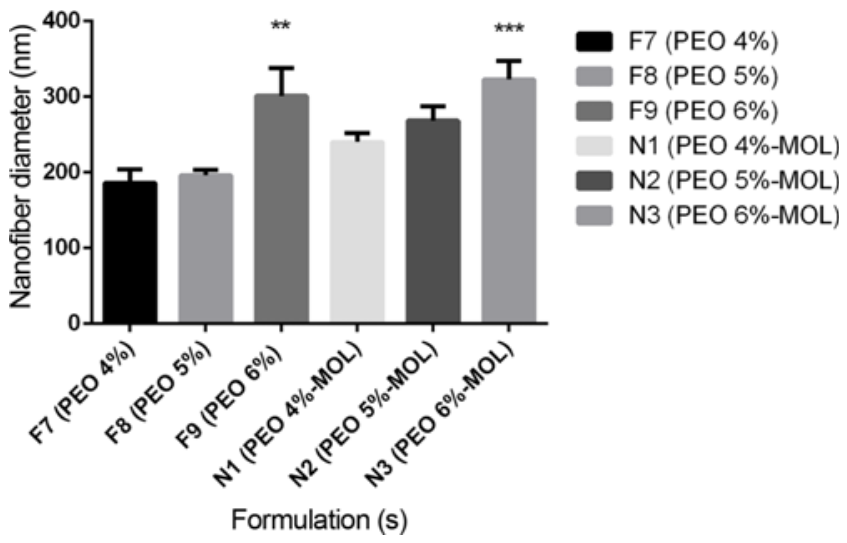

Figure 3. Nanofiber diameter ( $\mathrm{nm}$ ) of blank PEO nanofibers (F7F9) and MOL-loaded PEO nanofibers (N1-N3). The asterisks (**) represent significant difference $(* * \mathrm{p}<0.01)$ and $(* * *)$ represent very significant difference $(* * * p<0.001)$ to PEO $4 \%$ nanofiber.
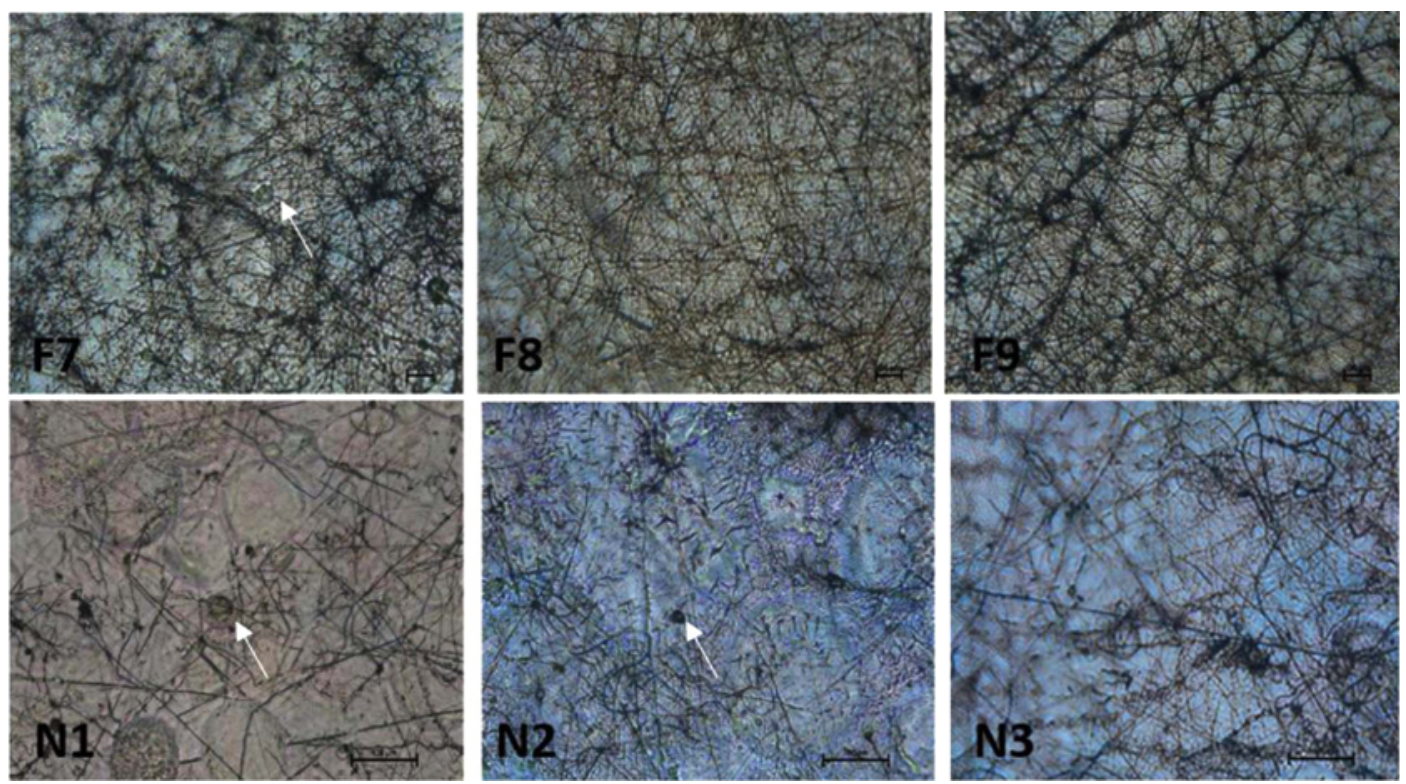

Figure 2. Microscopic images of pre-formulation nanofibers under a light microscope at x200 magnification. The clumps and beads could be seen in several formulations after electrospinning (labelled by white arrows). 


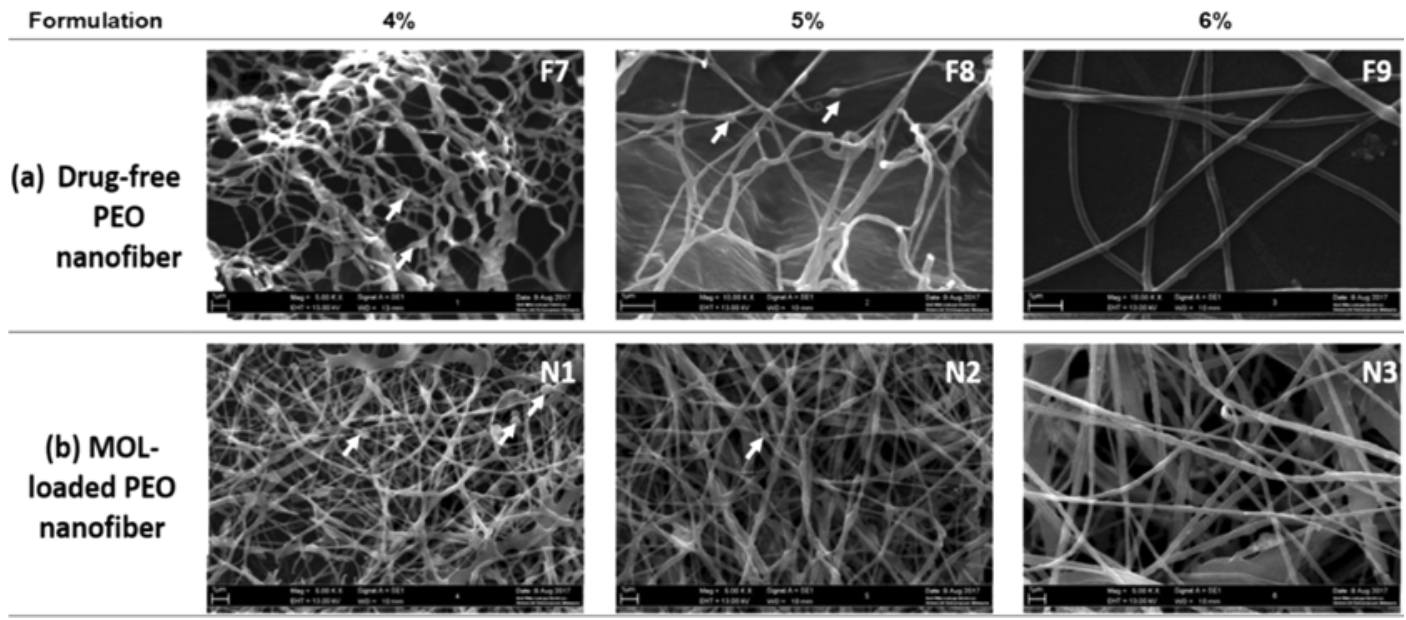

Figure 4. VPSEM images of (a) formulation F7-F9 (Blank PEO 4-6 \% w/v nanofiber), and (b) formulation N1-3 (MOL extract-loaded PEO 4-6 \% w/v nanofibers) at $5 \mathrm{k}$ and $10 \mathrm{kX}$ magnification. The clumps or beads in nanofibers indicated by the white arrows.

and MOL loaded PEO nanofiber's mesh distribution and fibre diameter were then compared via VPSEM. The VPSEM microscope images (Figure 4) showed that formulation F7 and F8 (PEO blank nanofibers) exhibited fishnet-like nanofibers with beads. While F9 nanofiber appeared in smooth uniform nanofiber without beads formation. Formulation F7, F8 and F9 appearance are in agreement with the previous microscope light observation (Figure 2). After MOL extract was loaded, N3 still exhibited uniform fibre without beads. The fibre diameter for each nanofiber formulations was measured via Image J. Figure 3 showed that F7 and F8 blank nanofiber exhibited fibre diameter $186.2 \pm 55.8 \mathrm{~nm}$ and $196.3 \pm 22.4 \mathrm{~nm}$ respectively with no significant differences. Whereas F9 blank nanofiber exhibited significantly increased in fibre diameter $301.2 \pm$ $116.3 \mathrm{~nm}(\mathrm{p}<0.01)$ compared to F7 nanofiber. The finding revealed that $\mathrm{PEO}$ concentrations were directly proportional to nanofiber diameters. As PEO concentration increased, the nanofiber diameter also increased as well. This observation could be attributed to high concentration of PEO increases the solution viscosity and chain entanglement between PEO polymeric chains [25]. Polymer chain entanglement is essential to form a continuous jet to produce a smooth uniform nanofiber. Hence the polymers which in low concentration or viscosity leading to insufficient chain entanglements and produces nanofiber in the form of beads or lumps on the fibrous strands [26,27]. After MOL extract blended with PEO for electrospinning, N1-N3 also found an increased in fibre diameter than the blank nanofibers. N1 and N2 nanofiber were found to possess fibre diameter $240 \pm 36.9 \mathrm{~nm}$ and $268.5 \pm 58.6 \mathrm{~nm}$ respectively with no significant difference. Whereas N3 nanofiber exhibited markedly increased in fibre diameter $322.7 \pm 78.02 \mathrm{~nm}$ with a very significant difference compared to $F 7(p<0.001)$. The increase in fibre diameter may attribute to addition of MOL extract into PEO nanofiber.

\section{Rheological Study}

The polymer solution viscosity has become one of the main factors affecting nanofiber morphology during the process of electrospinning. When the polymer solution concentration was too low, the fabricated nanofiber prone to produce discontinuous fibres. However, if polymer solution viscosity became too high, it will be causing obstruction to the ejection of polymer solution through electrospinning stainless needle. Thus, an optimal polymer solution viscosity is very crucial for electrospinning to ensure smooth uniform nanofibers. Here, the apparent viscosities at shear rate $300 \mathrm{~s}^{-1}$ of the polymer solution were measured and compared.

As illustrated in Figure 5a, the findings revealed that formulation polymer solution possess a higher apparent viscosity $(0.94 \pm 0.002 \mathrm{~Pa} \cdot \mathrm{s})$ than formulation $\mathrm{N} 1$ polymer solution $(0.611 \pm 0.008 \mathrm{~Pa} \cdot \mathrm{s})$ at shear rate $300 \mathrm{~s}^{-1}$. This might be attributed to the presence of MOL extract that had disrupted the chain entanglement of PEO polymers [27]. The low viscosities of F7 and N1 might also explains why N1 solution electrospun to nanofiber with beads as shown in VPSEM. Same trend was observed in F8/N2 and F9/N3. The F8 polymer solution exhibited higher apparent viscosity value $1.28 \pm 0.02 \mathrm{~Pa} \cdot \mathrm{s}$ than formulation N2 at $0.802 \pm 0.008$ $\mathrm{Pa} \cdot \mathrm{s}$ (Figure $5 \mathrm{~b}$ ). Whilst the apparent viscosity value for formulation F9 nanofiber showed markedly increased $(2.15 \pm 0.01)$ than formulation N3 $(1.38 \pm 0.01 \mathrm{~Pa} \cdot \mathrm{s})$. However, like $\mathrm{F} 7 / \mathrm{N} 1$, both $\mathrm{F} 8 / \mathrm{N} 2$ produced nanofibers with beads as shown in VPSEM. While N3/F9 produced uniform continuous nanofibrous strands in Figure 5c. Overall, it was observed that the viscosity of a solution affects the quality of the electrospun nanofibers. Here, given the electrospinning procedure established in our study, we could theorised that PEO polymer solution, with the addition of MOL extract must have apparent viscosity of above $1.30 \mathrm{~Pa} \cdot \mathrm{s}$ in order to 

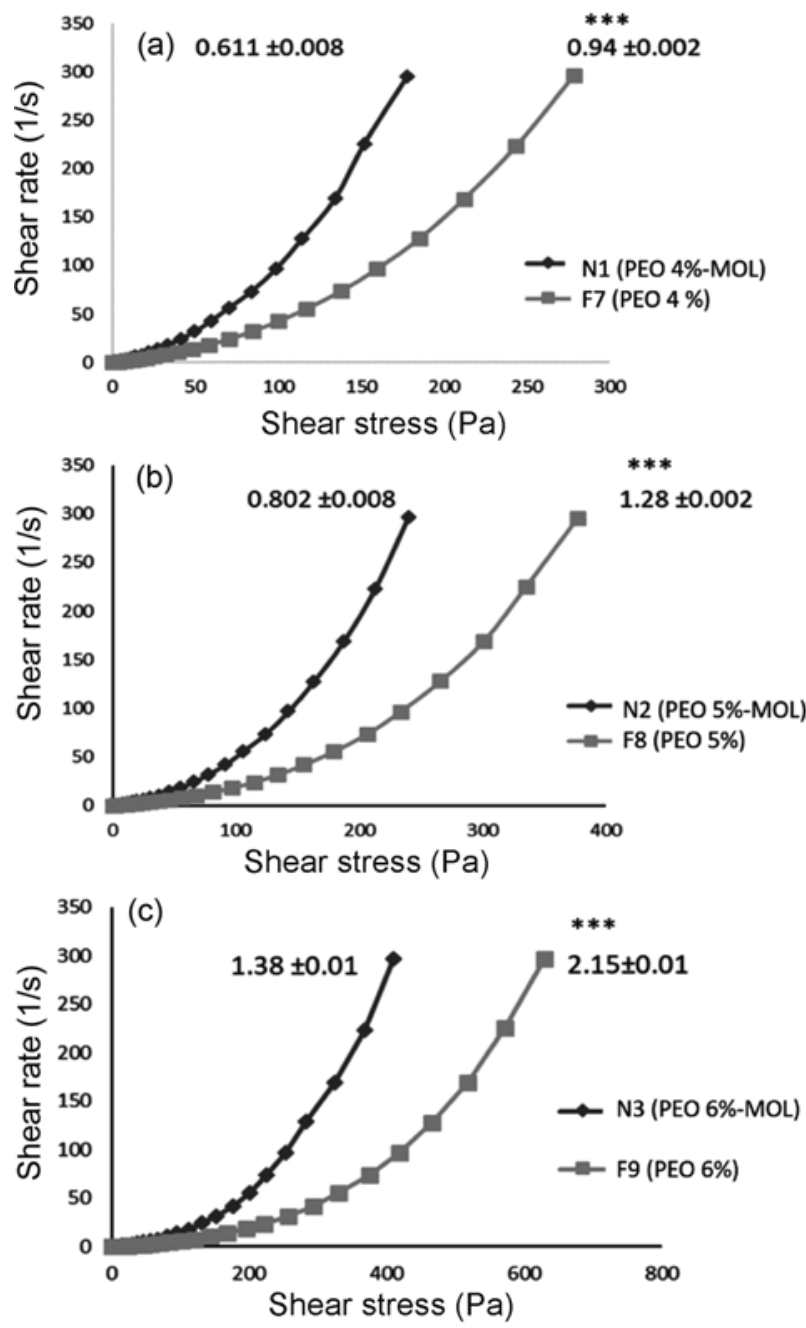

Figure 5. Apparent viscosities $(\mathrm{Pa} \cdot \mathrm{s})$ profile at a shear rate $300 \mathrm{~s}^{-1}$ of formulation (a) F7 (b) F8 (c) F9 blank PEO solution with respective N1-N3 (MOL loaded nanofiber) polymer solution before electrospinning. produce smooth and uniform electrospun nanofibers, while below $1.30 \mathrm{~Pa} \cdot \mathrm{s}$ would produce irregular nanofibers with beads.

\section{MOL-loaded Nanofiber-films}

After nanofiber characterisation using VPSEM and rheology studies, the most satisfactory MOL-loaded nanofiber (N3) were impregnated on SA-PC hydrocolloid film. The characterisation of film alone, blank nanofiber-film and MOL-loaded nanofiber-film were characterised by swelling ratio and moisture vapour transmission rate (MVTR).

\section{Swelling Ratio Study}

The swelling ratio study utilised the wound simulating fluid model to measure the swelling rate of the film alone, PEO nanofiber-film with and without MOL extracts at suppurating wound condition. The swelling property of wound dressing is very crucial as it controls the amounts of absorbed exudates and integrity of dressing for removal. As illustrated in Figure 6a, the data suggest that there was no significant difference in swelling ratio $(\mathrm{p}>0.05)$ between blank PEO nanofiber impregnated film $(39.3 \pm 2.6 \%)$ and MOL-loaded PEO nanofiber impregnated film $(44.9 \pm 2.9 \%)$ as compared to the vehicle SA-PC film $(50.4 \pm 0.9 \%)$ within $8 \mathrm{~h}$ of expansion study. The nanofiber impregnated films still maintained its integrity after $8 \mathrm{~h}$. The finding suggests that MOL loaded nanofiber impregnated film is indicated for light to the medium suppurating wound.

\section{Moisture Vapour Transmission Rate (MVTR)}

MVTR study is one of the important parameters to assess the gaseous exchange rate between wound bed and applied wound dressing. The estimated MVTR values for injured skin ranging between $278.4-5138.4 \mathrm{~g} / \mathrm{m}^{2} /$ day, in accordance to the previous study established by Bajpai et al. [28]. Thus, an ideal wound dressing should possess with optimal MVTR ranges in order to provide a moist wound-healing microenvironment by avoiding the risk of wound exudates build-
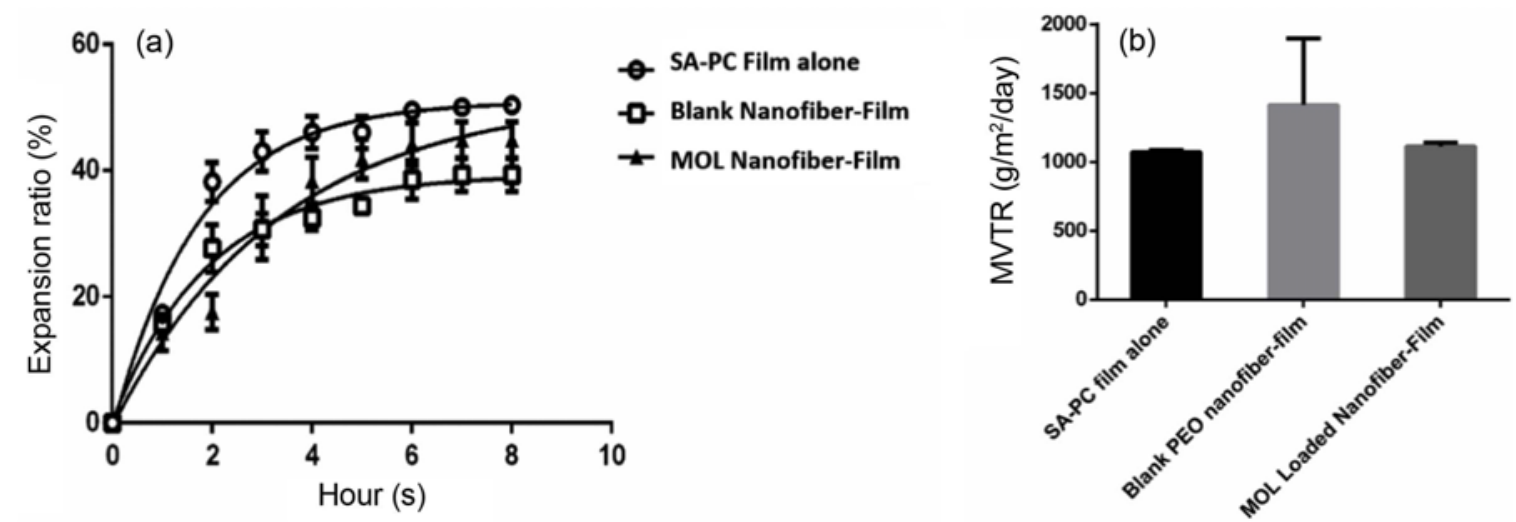

Figure 6. (a) Expansion study and (b) MVTR study of MOL aqueous extract-loaded PEO nanofiber-embedded film formulations $($ mean $\pm \mathrm{SD}, \mathrm{n}=3$ ). 
up or dehydration to wound bed. The MVTR result in Figure $6 \mathrm{~b}$ reported that there was no significant difference $(\mathrm{p}>0.05)$ found between blank PEO nanofiber impregnated film $\left(1415 \pm 483 \mathrm{~g} / \mathrm{m}^{2} /\right.$ day $)$ and MOL-loaded nanofiber impregnated film $\left(1115 \pm 28.1 \mathrm{~g} / \mathrm{m}^{2} /\right.$ day $)$ as compared to SA-PC vehicle film $\left(1073 \pm 15.7 \mathrm{~g} / \mathrm{m}^{2} /\right.$ day $)$. The overall finding suggests that MOL-loaded PEO nanofiber-film possess optimal MVTR for injured skin and supported the application for light to medium suppurating wounds.

\section{Optimisation of MOL Loaded Nanofibers-Hydrocolloid Film}

\section{Morphology of Nanofiber Impregnated Films}

Based on the nanofiber morphology under light microscope visualisation light microscope and VPSEM visualisation, formulation N3 (MOL-loaded PEO $6 \% \mathrm{w} / \mathrm{v}$ ) solution seems the most optimal concentration for electrospinning to fabricate a uniform smooth nanofiber without beads or droplets. It exhibited that the density of the nanofiber formed on the film surface increases as the electrospinning duration increases. Each of nanofiber impregnated films was then subjected to mechanical testing, moisture absorption study and drugrelease study to assess the effect of electrospinning duration.

\section{Thickness and Mechanical Properties}

The thickness of MOL loaded $6 \%$ PEO nanofiber-film at various electrospinning durations $(15,30$ and $60 \mathrm{~min})$ was compared in Figure $7 \mathrm{a}$. The data suggests that there was no
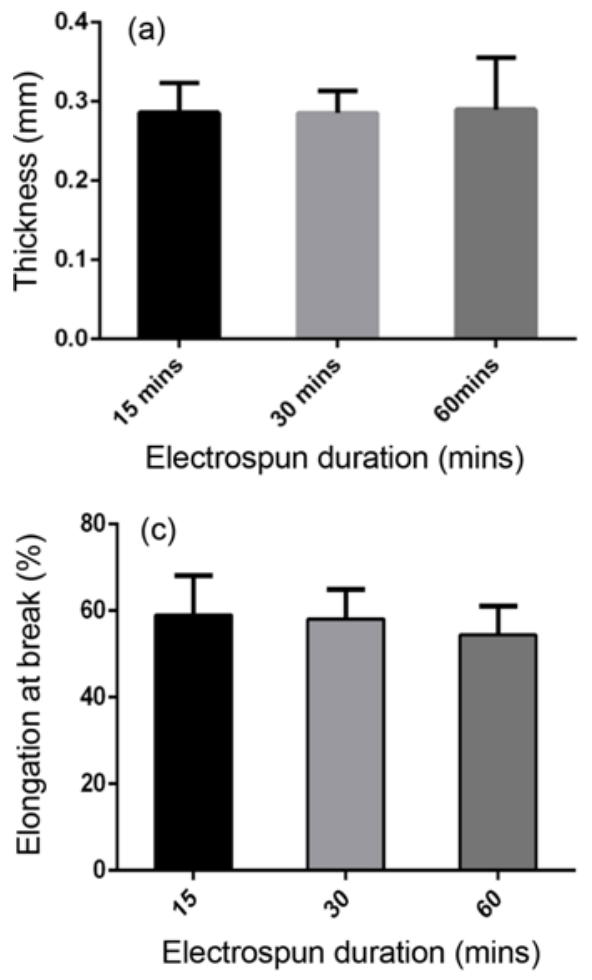

significant difference $(\mathrm{p}>0.05)$ in thickness of nanofiber-film of $15 \mathrm{~min}(0.286 \pm 0.038 \mathrm{~mm}), 30 \mathrm{~min}(0.286 \pm 0.028 \mathrm{~mm})$ and $60 \mathrm{~min}(0.289 \pm 0.065 \mathrm{~mm})$ electrospinning duration. This suggests that the electrospinning time between 15$60 \mathrm{~min}$ did not affect the nanofiber-film thickness significantly. The mechanical properties of the wound dressing such as tensile strength $(\mathrm{mPa})$ and elongation at break $(\%)$ are usually measured to test the flexibility and robustness of the wound dressing during application upon or removal from wound areas located at bendable areas such as knees or elbow. According to previously established wound healing study by Pereira et al. [29], the optimal tensile strength value for flexible skin areas ranged between 2.5 to $16 \mathrm{mPa}$ and $70 \%$ elongation at break (\%). As reported in Figure 7b, MOL-loaded PEO-nanofiber impregnated film with 15, 30 and $60 \mathrm{~min}$ electrospinning duration were exhibited tensile strength values at $2.70 \pm 0.71,2.72 \pm 0.64$ and $2.83 \pm 0.67 \mathrm{mPa}$ respectively with no significant difference $(\mathrm{p}>0.05)$ found among groups. Whereas MOL-loaded PEO-nanofiber impregnated film with 15, 30 and 60 min electrospinning durations have exhibited an elongation at break (\%) values at $59.01 \pm 9.18,58.08 \pm 6.89$ and $54.44 \pm 6.67 \%$ respectively with no significant difference $(\mathrm{p}>0.05)$ found among groups as shown in Figure $7 \mathrm{c}$. The finding suggests that the electrospinning duration does not cause the significant effect to the mechanical properties in term of thickness $(\mathrm{mm})$, tensile strength $(\mathrm{mPa})$ and elongation at break (\%) of
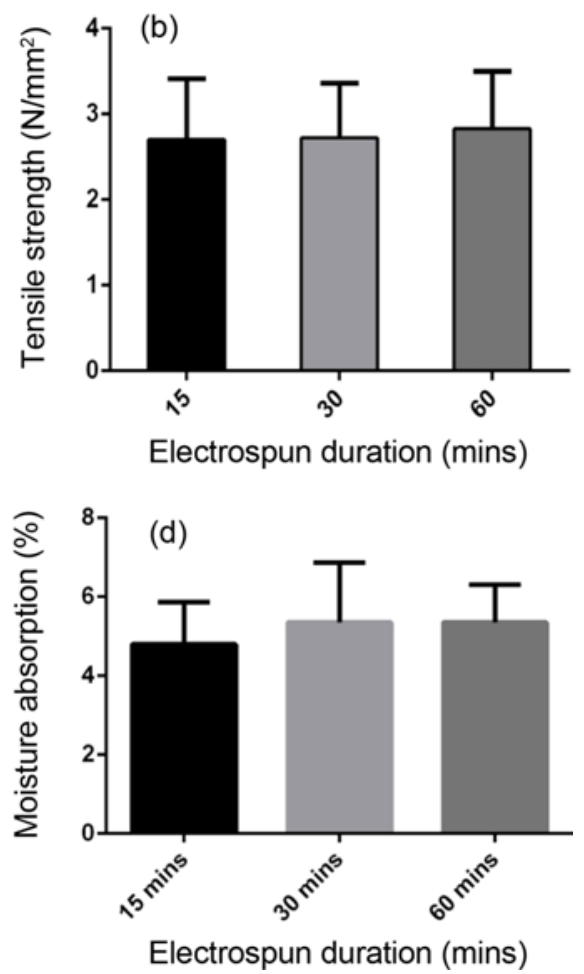

Figure 7. (a) Thickness, (b) tensile strength, (c) elongation at break, and (d) moisture absorption of MOL loaded $6 \%$ PEO nanofiberembedded film of various electrospinning durations (15, 30 and $60 \mathrm{~min})$ (mean $\pm \mathrm{SD}, \mathrm{n}=3$ ). 
nanofiber-films.

\section{Moisture Absorption Study}

The moisture absorption rates between MOL loaded nanofiber-films at various electrospinning durations (15, 30 and $60 \mathrm{~min}$ ) were compared. As illustrated in Figure $7 \mathrm{~d}$, the moisture absorption rates (\%) of each MOL loaded nanofiberimpregnated films $(15,30$ and $60 \mathrm{~min})$ were $4.79 \pm 1.08$, $5.35 \pm 1.51$ and $5.35 \pm 0.95 \%$ respectively with no significant difference among groups $(\mathrm{p}>0.05)$. The moisture absorption rate between $4.79-5.35 \%$ considered as low moisture absorption and this evidence supported that MOL loaded nanofiber-impregnated films are indicated for light to the medium suppurating wound.

In vitro Cumulative Drug Release Study Via Franz Diffusion Cells

The in vitro cumulative release of bioactive compounds (vicenin-2, chlorogenic acid, and rosmarinic acid) conducted
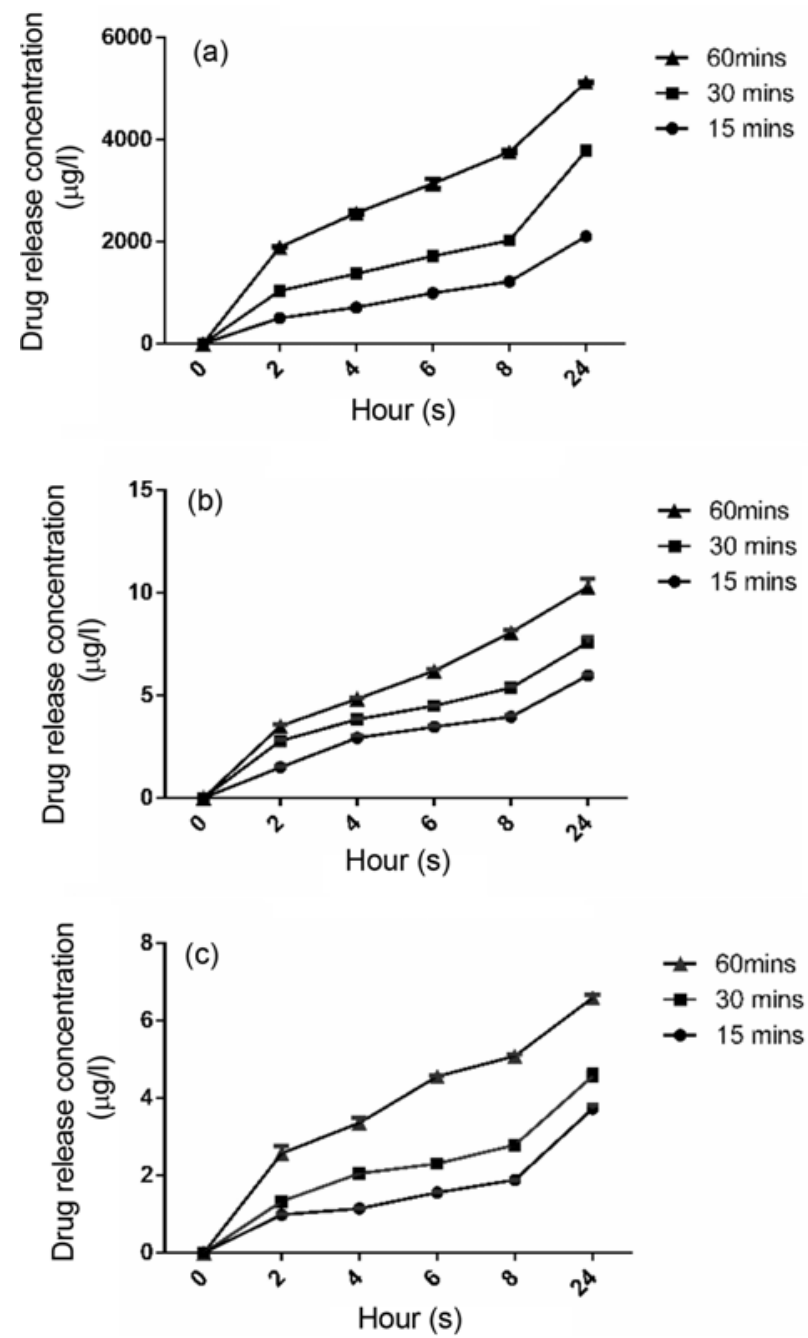

Figure 8. Cumulative release of bioactive compounds (a) vicenin2, (b) chlorogenic acid, and (c) rosmarinic acid in MOL extractloaded nanofiber embedded film (mean $\pm \mathrm{SD}, \mathrm{n}=3$ ). on nanofiber-film samples with various electrospinning durations (15, 30 and $60 \mathrm{~min}$ ) using Franz diffusion cells. The sample aliquots at first 8 th $\mathrm{h}$ and 24 th $\mathrm{h}$ of drug release were subjected to quantification using HPLC technique. Based on the overall release of bioactive compounds in Figure 8, it revealed that MOL extract loaded nanofiberfilms (15, 30 and $60 \mathrm{~min})$ exhibited a burst release after 2 nd $\mathrm{h}$ of drug release study. The nanofiber-films at 15-60 min electrospinning duration were released vicenin-2 at concentration between 504-1890 $\mu \mathrm{g} / \mathrm{l}$ respectively at $2 \mathrm{nd} \mathrm{h}$, followed by gradual increased between 2104-5124 $\mu \mathrm{g} / \mathrm{l}$ after $24 \mathrm{th} \mathrm{h}$. The amount of release of vicenin-2 had been remarkably increased as compared to the previous $1 \% \mathrm{MOL}$ hydrocolloid film $(102.07 \pm 1.089 \mu \mathrm{g} / l)$ at $2 \mathrm{nd} \mathrm{h}$ of cumulative release study. Besides that, the result also correlates that concentrations of released vicenin-2, chlorogenic acids and rosmarinic acids are increasing in electrospinning durationdependent manner. Hence 60 min-electrospun nanofiberfilm could release a higher amount of bioactives than 15 and $30 \mathrm{~min}$. A similar release trend also found in chlorogenic acid and rosmarinic acid. Based on overall findings, it suggests that the drug release of individual bioactives could be enhanced by the incorporation of $1 \% \mathrm{w} / \mathrm{v}$ MOL extract into nanofiber-film dressing.

\section{In Vitro Biocompatibility Assay}

In vitro biocompatibility assay was conducted using MTT assay on human dermal cell lines which are human dermal fibroblast (HDF) and epidermal keratinocytes (HEK) cell lines to assess the status of biocompatibility of MOL extract loaded nanofiber embedded films. As reported in Figure 9, the cell viability rates in HDF cell line which treated with MOL-loaded nanofiber embedded films under different electrospinning durations (15, 30 and $60 \mathrm{~min})$ were exhibited $221.1 \pm 17.1,224.8 \pm 7.5$ and $244.4 \pm 30.1 \%$ respectively with significant difference $(\mathrm{p}<0.001)$ as compared to the untreated control after $24 \mathrm{~h}$ study. Whereas PEO vehicle nanofiber exhibited viability rate at $98.92 \pm 10.49 \%$ with no significant difference $(p>0.05)$ to the untreated control. This finding suggests that the incorporation of MOL extract into PEO nanofiber would enhance the cell proliferation in HDF cell line.

Based on the cell viability rate in HEK cell line reported in Figure 9, the finding revealed that the HEK cell viability rates treated with MOL loaded nanofiber embedded films $(15,30$ and $60 \mathrm{~min})$ were $443.8 \pm 10.5,488.7 \pm 38.1$ and $502.9 \pm 29.3 \%$ respectively. This evidence suggests that the incorporation of MOL extract into nanofiber had markedly increased cell proliferation beyond $400 \%$ in HEK cell line as compared to the untreated control $(\mathrm{p}<0.001)$. As seen in both HDF and HEK cell lines, the cell viability rate found to be increased as the electrospinning duration increases. This phenomenon could be explained that nanofiber structure mimics the extracellular matrix (ECM) as a supporting matrix for cell attachment while MOL extract helps in 


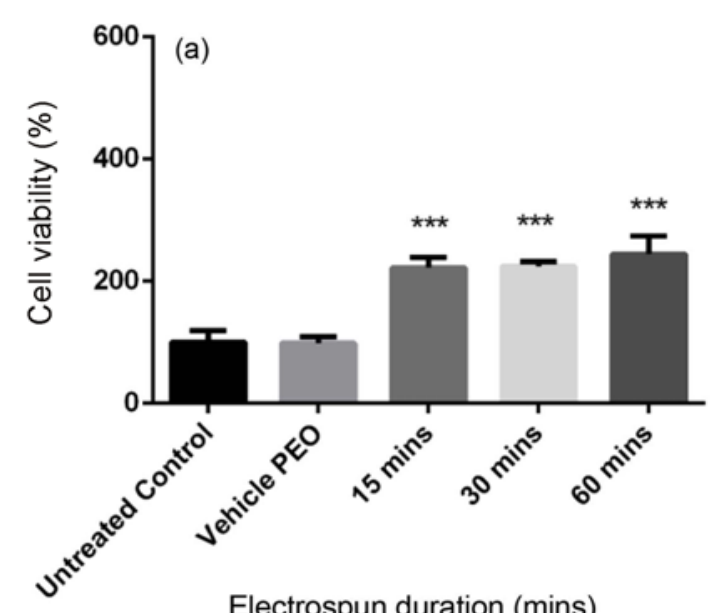

Electrospun duration (mins)

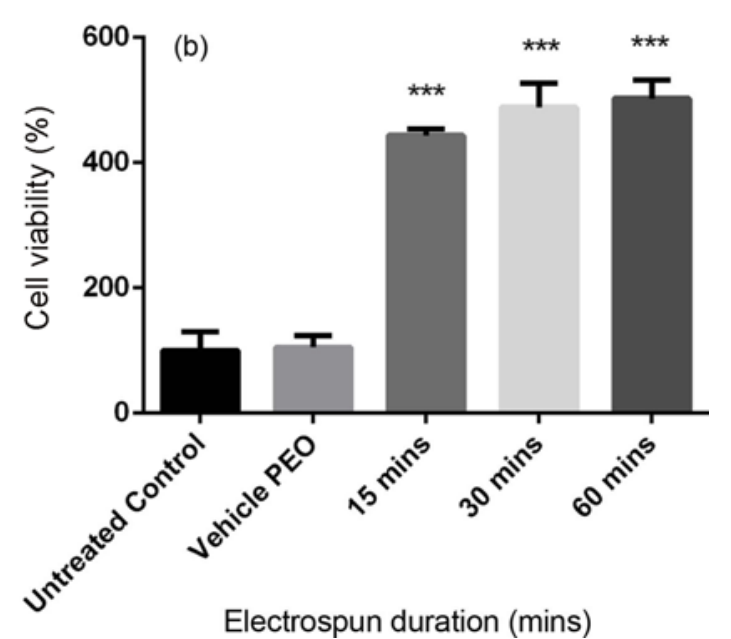

Figure 9. (a) Human dermal fibroblast (HDF) and (b) human epidermal keratinocytes (HEK) cell proliferation study on MOL aqueous extract-loaded PEO nanofiber-embedded film formulations (mean $\pm \mathrm{SD}, \mathrm{n}=3$ ). The asterisks $(*)$ represent significant difference $(\mathrm{p}<0.05)$ to untreated control (normal media).

boosting cell proliferation. In accordance with the ISO 10993-5:2009 guideline, the cell viability rate beyond $80 \%$ considered good cell biocompatibility and non-toxic to host cells [30]. Hence these findings supported that MOL-loaded nanofiber impregnated films at various electrospinning durations are non-toxic and showed excellent biocompatibility to human dermal cells.

\section{Conclusion}

In this study, a novel PEO nanofiber impregnated hydrocolloid film loaded with MOL extract had successfully developed via the electrospinning technique. The MOL extract also successfully blended into PEO nanofiber as a wound healing agent with its hydrocolloid film backing layer to make dressing application lasts longer. This could provide new insight into developing nanofiber-based wound dressing for potential delayed chronic wound treatment.

\section{Acknowledgements}

The authors gratefully acknowledged the financial support provided by NRGS grant (NH1014 S016) from the Ministry of Agriculture (MoA) Malaysia and also thanks to The Universiti Kebangsaan Malaysia (UKM) KL campus and Tissue Engineering Centre (TEC), PPUKM for the research facility support.

\section{References}

1. Federation ID, IDF Diabetes Atlas., 7, 12 (2019).

2. P. Kamble, B. Sadarani, A. Majumdar, and S. Bhullar, $J$. Drug Deliv. Sci. Technol., 41, 124 (2017).

3. L. Lin, Y. Gu, and H. Cui, Food Packaging and Shelf Life, 19, 86 (2019).

4. H. Cui, M. Bai, M. M. A. Rashed, and L. Lin, Int. J. Food Microbiol., 266, 69 (2018).

5. H. Cui, C. Zhang, C. Li, and L. Lin, Ind. Crops Prod., 140, 111739 (2019).

6. M. Hashmi, S. Ullah, and I. S. Kim, Current Res. Biotechnol., 1, 1 (2019).

7. M. Hromadka, J. B. Collins, C. Reed, L. Han, K. K. Kolappa, B. A. Cairns, T. Andrady, and J. A. van Aalst, J. Burn Care Res., 29, 695 (2008).

8. S. Ullah, M. Hashmi, M. Q. Khan, D. Kharaghani, Y. Saito, T. Yamamoto, and I. S. Kim, RSC Adv., 9, 268 (2019).

9. S. Jaganathan, A. Balaji, A. F. Ismail, and R. Rajasekar, Int. J. Nanomed., 11, 4339 (2016).

10. D. Kharaghani, P. Gitigard, H. Ohtani, K. O. Kim, S. Ullah, Y. Saito, M. Q. Khan, and I. S. Kim, Scientific Reports, 9, 12640 (2019).

11. S. Ullah, M. Hashmi, D. Kharaghani, M. Q. Khan, Y. Saito, T. Yamamoto, J. Lee, and I. S. Kim, Int. J. Nanomed., 14, 2693 (2019).

12. M. Q. Khan, D. Kharaghani, Sanaullah, A. Shahzad, Y. Saito, T. Yamamoto, H. Ogasawara, and I. S. Kim, Polymer Testing, 74, 39 (2019).

13. C.-M. Lin, Y.-C. Chang, L.-C. Cheng, C.-H. Liu, S. C. Chang, T.-Y. Hsien, D.-M. Wang, and H.-J. Hsieh, Cellulose, 27, 2651 (2020).

14. F. Anwar, S. Latif, M. Ashraf, and A. H. Gilani, Phytother Res., 21, 17 (2007).

15. E. J. Choi, T. Debnath, Y. Tang, Y. B. Ryu, S. H. Moon, and E. K. Kim, Biomed Pharmacother, 84, 870 (2016).

16. N. Z. Abd Rani, K. Husain, and E. Kumolosasi, Frontiers in Pharmacology, 9, 108 (2018).

17. B. S. Rathi, S. L. Bodhankar, and A. M. Baheti, Indian J. Exp. Biol., 44, 898 (2006). 
18. A. A. Muhammad, S. Fakurazi, P. Arulselvan, C. P. See, and F. Abas, Drug Des. Devel. Ther, 10, 1715 (2016).

19. C. Y. Chin, J. Jalil, P. Y. Ng, and S. F. Ng, J. Ethnopharmacol., 212, 188 (2018).

20. C. Y. Chin, P. Y. Ng, and S. F. Ng, Drug Deliv. Transl. Res., 9, 453 (2019).

21. A.-W. Lim, P.-Y. Ng, N. Chieng, and S.-F. Ng, J. Drug Deliv. Sci. Technol., 54, 101329 (2019).

22. M. Krstić, M. Radojević, D. Stojanović, V. Radojević, P. Uskoković, and S. Ibrić, Eur. J. Pharm. Sci., 101, 160 (2017).

23. H. E. Thu, M. H. Zulfakar, and S. F. Ng, Int. J. Pharm., 434, 375 (2012).

24. K. H. Matthews, H. N. E. Stevens, A. D. Auffret, M. J. Humphrey, and G. M. Eccleston, Int. J. Pharm., 289, 51
(2005).

25. S. C. Moon, B. Y. Ryu, J. K. Choi, B. W. Jo, and R. J. Farris, Polym. Eng. Sci., 49, 52 (2009).

26. A. Polini and F. Yang in "Nanofiber Composites for Biomedical Applications", p.97, Woodhead Publishing, 2017.

27. C. A. Bonino, M. D. Krebs, C. D. Saquing, S. I. Jeong, K. L. Shearer, E. Alsberg, and S. A. Khan, Carbohydr. Polym., 85, 111 (2011).

28. M. Bajpai, S. K. Bajpai, and D. Gautam, J. Appl. Chem., 2014, 9 (2014).

29. R. Pereira, A. Carvalho, D. C. Vaz, M. H. Gil, A. Mendes, and P. Bártolo, Int. J. Biol. Macromol., 52, 221 (2013).

30. ISO 10993-5: 2009, "Biological Evaluation of Medical Devices-Part 5: Tests for in vitro Cytotoxicity”, 2009. 